\title{
Response versus scan-angle corrections for MODIS reflective solar bands using deep convective clouds
}

\author{
Rajendra Bhatt*a, Amit Angal ${ }^{\mathrm{a}}$, David R. Doelling ${ }^{\mathrm{b}}$, Xiaoxiong Xiong ${ }^{\mathrm{c}}$, Aisheng $\mathrm{Wu}^{\mathrm{a}}$, Conor O. \\ Haney $^{\mathrm{a}}$, Benjamin R. Scarino ${ }^{\mathrm{a}}$, Arun Gopalan ${ }^{\mathrm{a}}$, \\ ${ }^{a}$ SSAI, One Enterprise Pkwy Ste 200, Hampton, VA 23666 USA \\ bNASA Langley Research Center, 21 Langley Blvd MS 420, Hampton, VA 23681 USA \\ 'NASA Goddard Space Flight Center, 8800 Greenbelt Rd, MS 618, Greenbelt, MD 20771 USA \\ *rajendra.bhatt@nasa.gov; phone 1 (757) 9511656
}

\begin{abstract}
The absolute radiometric calibration of the reflective solar bands (RSBs) of Aqua- and Terra-MODIS is performed using on-board calibrators. A solar diffuser (SD) panel along with a solar diffuser stability monitor (SDSM) system, which tracks the performance of the SD over time, provides the absolute reference for calibrating the MODIS sensors. MODIS also views the moon and deep space through its space view (SV) port for lunar-based calibration and computing the zero input radiance, respectively. The MODIS instrument views the Earth's surface through a two-sided scan mirror, whose reflectance is a function of angle of incidence (AOI) and is described by response versus scan-angle (RVS). The RVS for both MODIS instruments was characterized prior to launch. MODIS also views the SD and the moon at two different assigned RVS positions. There is sufficient evidence that the RVS is changing on orbit over time and as a function of wavelength. The SD and lunar observation scans can only track the RVS variation at two RVS positions. Consequently, the MODIS Characterization Support Team (MCST) developed enhanced approaches that supplement the onboard calibrator measurements with responses from pseudo-invariant desert sites. This approach has been implemented in Level 1B (L1B) Collection 6 (C6) for selected short-wavelength bands. This paper presents an alternative approach of characterizing the mirror RVS to derive the time-dependent RVS correction factors for MODIS RSBs using tropical deep convective cloud (DCC) targets. An initial assessment of the DCC response from Aqua-MODIS band 1 C6 data indicates evidence of RVS artifacts, which are not uniform across the scans and are more prevalent in the left side Earthview scans.
\end{abstract}

Keywords: MODIS, RVS, mirror response, DCC

\section{INTRODUCTION}

The Clouds and the Earth's Radiant Energy System (CERES) project relies on the long-term radiometric accuracy and stability of Aqua-MODIS band 1 radiances for consistent calibration of multiple geostationary (GEO) satellite imagers that are used to fill in the gap between the CERES observed fluxes from the sun-synchronous Terra (10:30 AM) and Aqua (1:30 PM) orbits ${ }^{1}$. The Aqua-MODIS calibration is transferred to the GEO imagers via ray-matching co-incident, co-angled, and co-located regions imaged by the two sensors over an equatorial domain centered at the GEO subsatellite point ${ }^{2}$. This infers that any MODIS calibration drift manifests itself in the GEO ray-matched calibration. The CERES project verifies the MODIS band 1 stability using invariant desert, polar ice, and deep convective cloud (DCC) targets $^{3,4}$. The spatial domain for GEO-to-MODIS ray matching calibration extends from $\pm 30^{\circ}$ longitude and $\pm 20^{\circ}$ latitude from the GEO sub-satellite point. The large size of the domain is justified because it maximizes the number of coincident matches by including the MODIS observations from a wider range of view angles. This requires that the MODIS calibration is consistent across scan angle, and therefore, necessitates the CERES project to monitor any unaccounted MODIS scan angle dependency over time.

*rajendra.bhatt@nasa.gov; phone 1 (757) 9511656 
MODIS is a cross-track scanning radiometer with a two-sided scan mirror that images the Earth with a total angular field of view of $110^{\circ}$, extending $55^{\circ}$ on either side of the nadir ${ }^{5}$. The reflectance of the scan-mirror is not uniform and is a function of angle of incidence (AOI) as well as wavelength. This feature of the scan-mirror is known as response versus scan-angle (RVS) and was characterized for each MODIS wavebands prior to launch ${ }^{4}$. However, the RVS characteristic of MODIS changes on-orbit ${ }^{6}$ and, therefore, it must be tracked over time to ensure high data quality in the MODIS products. In addition, lack of correction for any RVS artifact in MODIS will also impair GEO derived fluxes in the CERES products because the GEO imagers are calibrated using MODIS radiances acquired over all AOIs.

The MODIS Characterization and Support Team (MCST) has implemented several approaches ${ }^{6,7}$ to track the MODIS RVS changes on orbit and applied correction for it to improve the radiometric quality of Collection 6 (C6) data. The reflective solar bands (RSBs) of both Aqua- and Terra-MODIS are radiometrically calibrated in orbit using a set of onboard calibrators. This set consists of a solar diffuser (SD) panel along with a solar diffuser stability monitor (SDSM) system, which tracks the performance of the SD over time, thereby providing an absolute reference for calibrating the MODIS sensors on the reflectance scale. The spatial and spectral characterizations (RSBs only) are monitored onboard with the spectroradiometric calibration assembly (SRCA) ${ }^{5}$. MODIS also collects lunar observations every month through its space view (SV) port for lunar-based stability assessment of the RSB calibration. The scan-mirror AOIs for SD and SV positions are fixed at $50.2^{\circ}$ and $11.2^{\circ}$, respectively ${ }^{6}$. The MSCT utilizes the SD and lunar observation scans to track the scan-mirror response over time at these two AOIs. Because the AOI for Earth view (EV) observations varies from $10.5^{\circ}$ to $65.5^{\circ}$, the measurements from the onboard calibrators are supplemented by EV response from pseudo-invariant calibration sites (PICS) to facilitate the on-orbit RVS characterization at other AOIs ${ }^{6,7}$. Because ground sites have fixed geo-locations, observations from multiple PICS are required to characterize RVS at all possible AOIs. Although the PICS approach has been successfully used in monitoring the temporal stability of numerous satellite sensors for many years, its usage for RVS trending involves higher uncertainty as it requires all of the PICS imaged by the MODIS sensors at different AOIs be radiometrically stable during the same period of time.

In this paper, we propose a novel approach of characterizing the MODIS RVS using tropical deep convective clouds (DCC). The baseline method is similar to what is described in the GSICS DCC ATBD 6 and by Doelling et al. ${ }^{9}$, except that the DCC PDFs and temporal trends are computed at different sets of AOIs so that scan-specific DCC responses can be computed. The method is applied to Aqua-MODIS band 1 and the observed RVS drifts are presented in the paper.

\section{METHODOLOGY}

Previous studies ${ }^{9,10,11,12,13,14}$ have shown that DCCs are an excellent invariant Earth target for vicarious calibration of satellite reflective solar bands. They are located at tropopause height, where the impact of water vapor absorption is minimal, have the highest signal-to-noise ratio, and have nearly lambertian reflectance spectra in the visible range. They are distributed globally over the tropics, and therefore, are viewed by all satellites. Being the coldest Earth targets, DCCs can be identified using a simple IR temperature threshold. In this study, MODIS DCC pixels are selected globally over the tropics within $\pm 20^{\circ}$ latitude. DCC radiance pixels are identified within the domain using an IR threshold of $205 \mathrm{~K}$ and are further passed through spatial homogeneity tests, as described in the GSICS DCC ATBD ${ }^{8}$, to get rid of optically thin clouds. In general, a DCC radiance pixel is considered valid if it meets the following criteria: BT $11 \mu \mathrm{m}<205 \mathrm{~K}$, $\mathrm{SZA}<40^{\circ}, \mathrm{VZA}<40^{\circ}, 10^{\circ}<\mathrm{AZA}<170^{\circ}, \sigma(\mathrm{BT} 11 \mu \mathrm{m})<1.0 \mathrm{~K}$, and $\sigma(\mathrm{VIS})<3 \%$. The angular normalization in the MODIS DCC pixels is performed using the angular distribution model (ADM) by Hu et al ${ }^{14}$. The statistical mode of the normalized DCC radiances, compiled into monthly probability distribution functions (PDFs), is tracked over time to monitor a sensor's radiometric stability.

Tests for any RVS drift in the MODIS sensors are performed by grouping the DCC pixels into multiple sets of AOIs along the MODIS scans on both sides of the nadir view. A $\pm 55^{\circ}$ MODIS scan achieves a $2330-\mathrm{km}$ swath and consists of 1354 frames, which can be linearly mapped to AOIs ranging from $10.5^{\circ}$ to $65.5^{\circ}$. Figure 1 a shows the scan-mirror AOIs and corresponding frame numbers for onboard calibrators and different EV scans. The AOIs for the SD, SRCA, and SV port are $50.3^{\circ}, 38.2^{\circ}$, and $11.2^{\circ}$, which correspond to frames 980,682 , and 18 , respectively. The nadir Earth view is covered in frame 678. Therefore, all left-side scan DCC pixels lie between frames 1 through 678, whereas the rest of the DCC pixels acquired by the right side of the MODIS scan are covered by frames 679 through 1354. The monthly PDFs are computed separately for the groups of DCC pixels so formed, and their modes are regressed over time to analyze any drift in the mirror response between the different scan positions. The monthly PDFs computed for Aqua-MODIS band 1 using the DCC pixels, acquired in the frames that are on the left and right sides of EV nadir, are shown in Figure 1b and $1 c$, respectively. It is notable that the PDFs computed from DCC pixels acquired in frames 679-1354 are more stable 
than those from frames 1-678. The peaks of the PDFs have lined up very well in the former case, whereas, in the latter case, they have shifted slightly left over time, thereby suggesting RVS change in the Aqua-MODIS band 1 mirror response in the latest C6 calibration. A more accurate estimate of the temporal RVS drift is made by performing a linear regression to the time-series of the monthly PDF modes computed for each set of frames. This is illustrated in more detail in the next section.
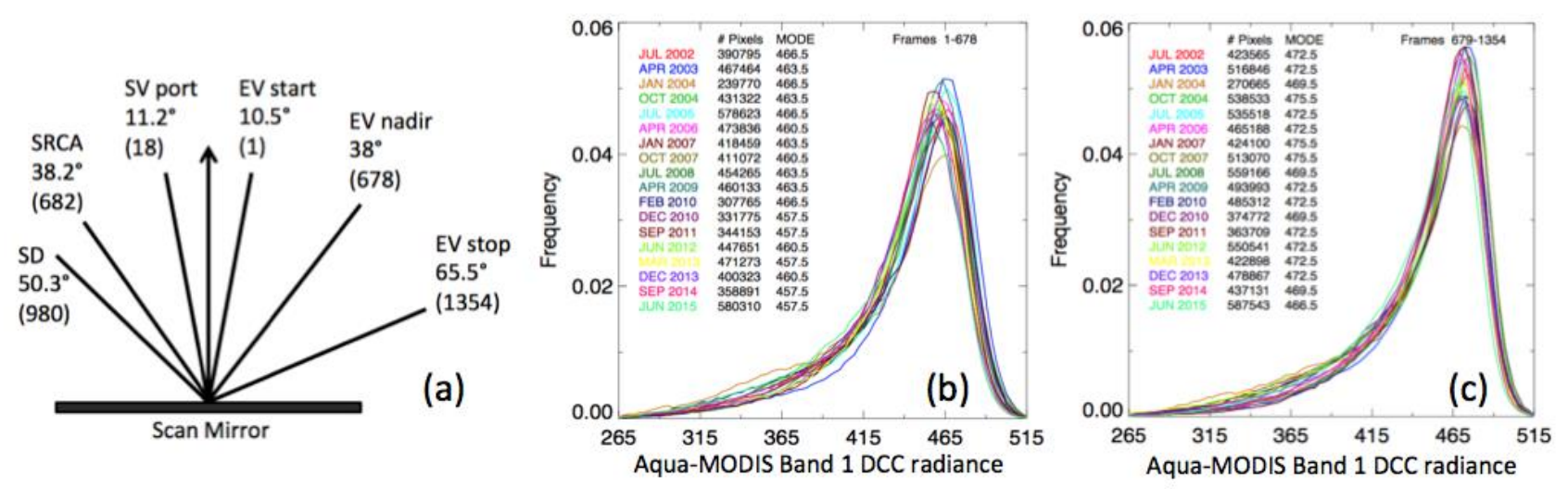

Figure 1. (a) The AOIs and frame numbers for onboard calibrators and EV positions, (b) monthly DCC PDF modes for left side scans (frames 1-678), and (c) same for right-side scans (frames 679-1354), derived using globally acquired Aqua-MODIS band 1 DCC pixels. Number of DCC identified MODIS pixels and the DCC PDF ADM corrected mode radiance is also given for certain months on the left side of the plot.

MODIS sensor responses are also computed at multiple AOIs over the Libya-1 desert PICS in order to independently validate the DCC-based RVS drifts. The region-of-interest (ROI) chosen over Libya- 1 was a $0.5^{\circ} \mathrm{x} 0.5^{\circ}$ box centered at $24.42^{\circ} \mathrm{N}$ latitude and $13.35^{\circ} \mathrm{E}$ longitude. The top-of-atmosphere MODIS reflectances acquired over all view angles over Libya-1 are used for this purpose. The clear-sky conditions are identified through a spatial homogeneity test as described by Bhatt et $\mathrm{al}^{14}$. The MODIS TOA clear-sky reflectances are then grouped by multiple sets of AOIs (or frame). An anisotropic correction is implemented to each set of data using a semi-empirical approach based on a linear combination of two kernel functions as described by Roujean et $\mathrm{al}^{15}$. Owing to the fact that the Aqua-MODIS band 1 has RVS drift over the long term, the coefficients of the kernel functions for Libya- 1 are computed using the MODIS observations during the first 5 years of operation, which is assumed to be the most stable period of MODIS calibration. The anisotropically corrected reflectances are then averaged on a monthly basis and are regressed over time for each set of frames to compute the desert RVS trends.

\section{RESULTS AND DISCUSSION}

This study performs RVS analysis by dividing the MODIS scans equally into four scan positions, which are defined by frame ranges 1-338, 339-678, 679-1016, and 1017-1354. The Aqua-MODIS band 1 DCC response trends computed for these scan positions are shown in Figure 2. The linear temporal regression statistics for all four cases are also shown. The PDF mode discretization is due the histogram bin size to identify the mode bin. Smaller bin size may track the degradation more accurately, however, may also introduce noise due to insufficient sampling across bins. The results show the RVS change in Aqua-MODIS band 1 is not uniform across the scans. The left side scans, especially between frames 1-338, exhibit a much larger RVS artifact compared to the right side scans. The trends show that the AquaMODIS band 1 RVS drift is $-2.2 \%$, on average, between frames $1-338,-1.6 \%$ between frames $339-678,-0.9 \%$ between frames 679-1016, and $-0.6 \%$ in frames 1017-1354, during 2002 to 2015. The lesser magnitude of RVS change in the right side scans proves that the on-orbit RVS tracking based on SD/SDSM observations, which are acquired at frame 980, is effective in maintaining the RVS stability for the entire right side scan within $1 \%$. On the other hand, the lunar observations performed through the SV port might have tracked the RVS at frame 18, but are inadequate to accurately characterize the on-orbit RVS drift in its nearby frames.

Alternatively, the Aqua-MODIS band 1 RVS trends are also computed from the Libya-1 observations for the same four sets of frames that were used in the DCC approach. The TOA reflectances are normalized to adjust the trend lines to 1 at the start of the timelines and are shown in Figure 3. The RVS changes computed from the desert are $-1.9 \%,-1.2 \%$, - 
$0.6 \%$, and $-0.6 \%$ for frames 1-338, 339-678, 679-1016, and 1017-1354, respectively. Because atmospheric effects impair the TOA measurements over Libya-1, the standard error of regression for the desert data is much higher compared to that for the DCC data. An annual seasonal cycle is also apparent in the Libya-1 TOA reflectances, particularly at high view angles, such as in frames 1-338 and 1017-1354. This inter-annual variability can be attributed to 1) the seasonal variability in the atmosphere above Libya-1, which has much higher impact on observations made at oblique view angles because of longer path-length, and 2) the surface bidirectional effects from the desert sand dunes, which cast shadows differently with changing sun-elevation - effects of which are not adequately captured by the kernel-driven bidirectional model used in this study. Despite the higher temporal variability in the desert data, the RVS drifts computed from Libya1 demonstrate good agreement with the DCC results.

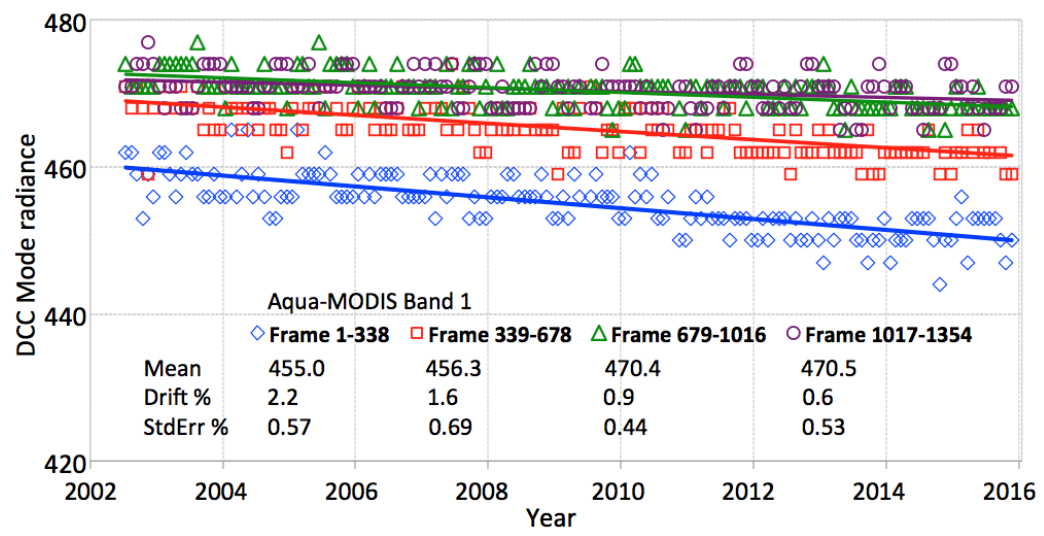

Figure 2. The DCC-based RVS trends computed for Aqua-MODIS band 1 frame 1-338 (Blue diamonds), 339-678 (Red squares), 679-1016 (Green triangles), and 1017-1354 (Purple circles).
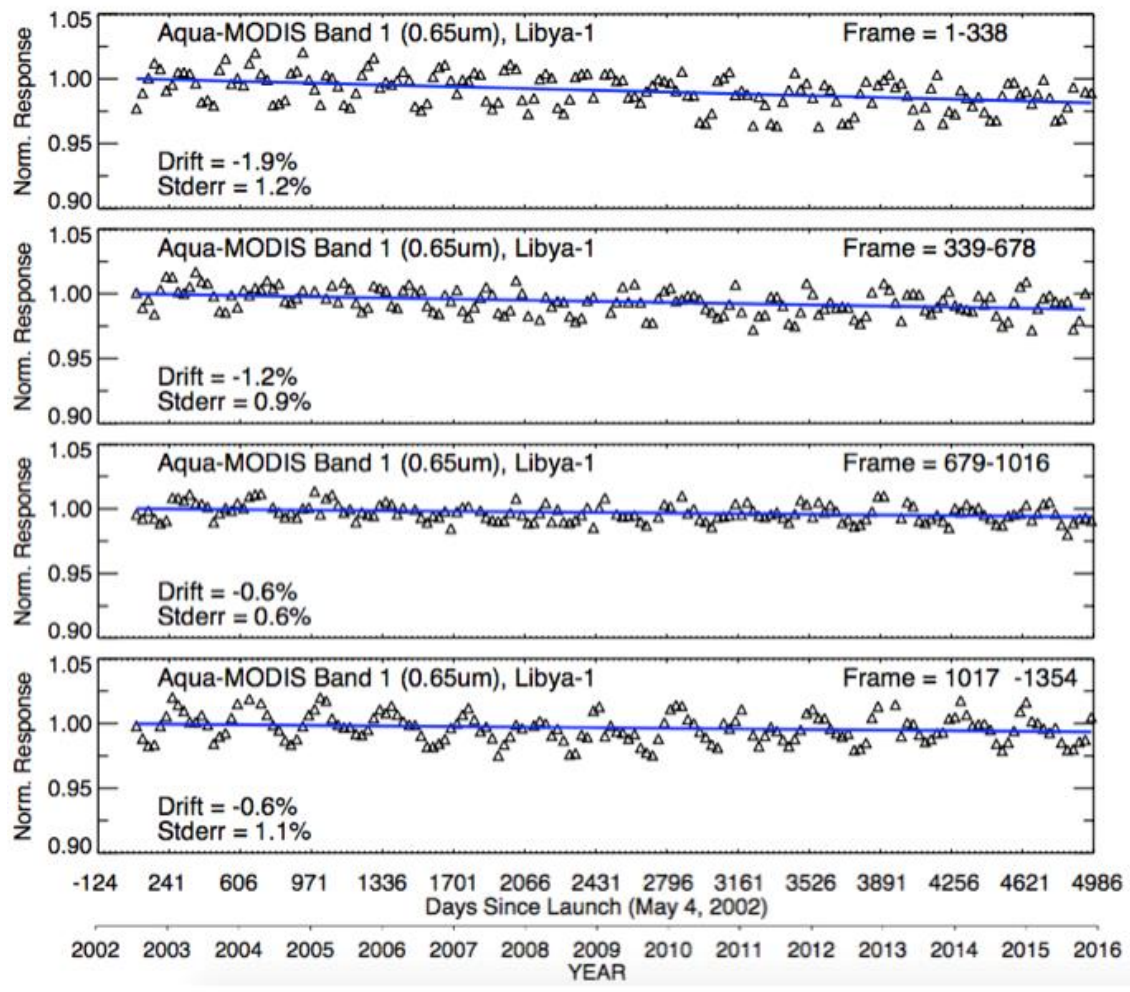

Figure 3. The Aqua-MODIS band 1 RVS trends computed for frames 1-338, 339-678, 679-1016, and 1017-1354 using clear-sky observations over Libya-1. 
Time-dependent RVS correction factors were derived for the four sets of frames on a monthly basis using the DCC RVS linear regression slopes and offsets. These correction factors are then applied to the desert reflectance time series in order to get rid of the RVS artifact. The new trend magnitudes for all four scan positions are less than $0.5 \%$, thereby proving the effectiveness of the DCC-based RVS corrections.

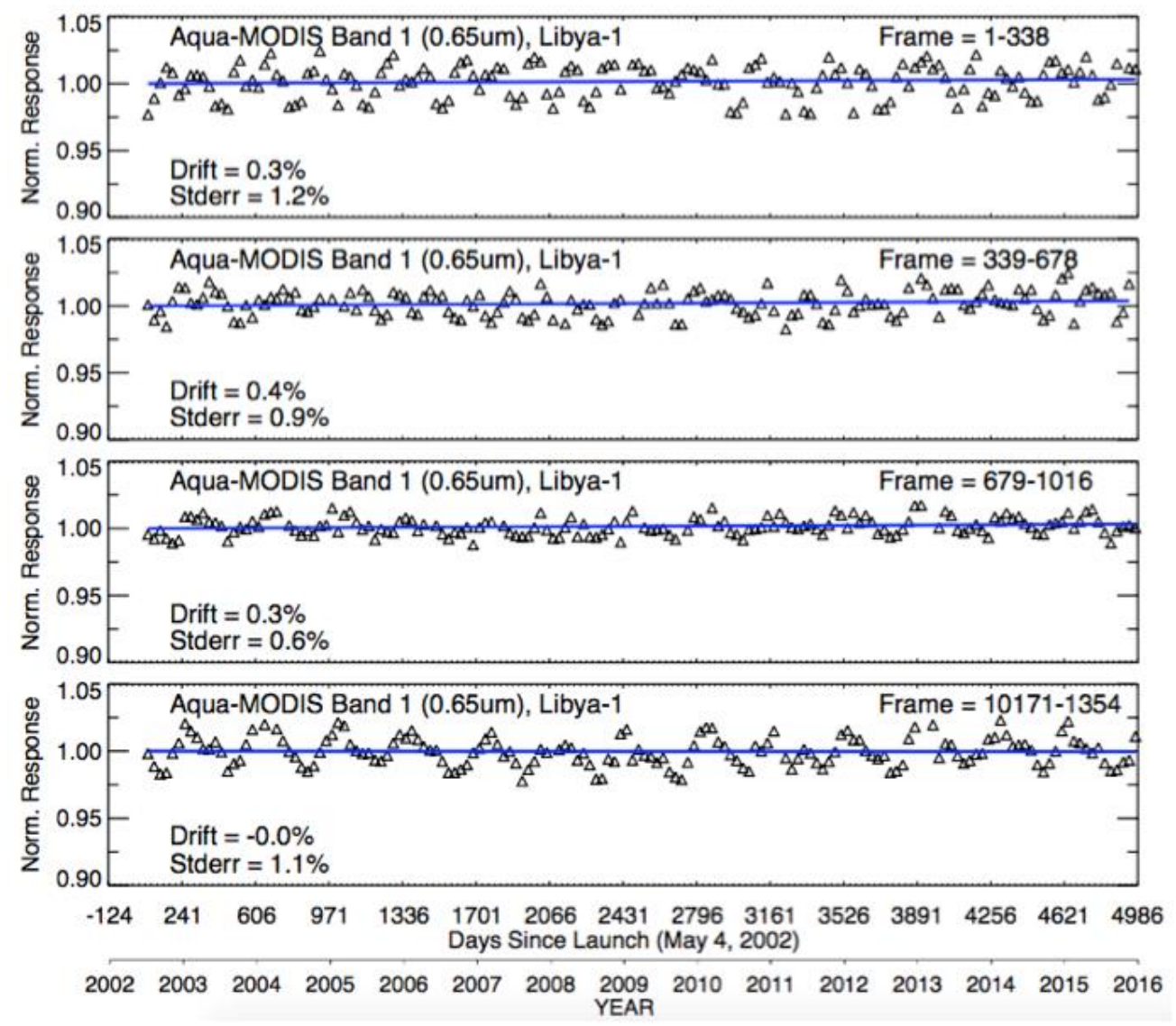

Figure 4. Same as Figure 3 but after applying DCC-based RVS corrections to the desert data.

\section{CONCLUSIONS}

A novel method of characterizing on-orbit RVS changes in MODIS RSBs using DCC is discussed in this paper. The method utilizes globally acquired tropical DCC pixels, which are grouped by different sets of MODIS frames, and tracks monthly DCC mode responses at the specified frames to compute the temporal changes in RVS. Initial results have shown that the Aqua-MODIS band 1 has been considerably impaired by RVS, which is more predominant in the left side scans. The magnitude of the RVS changes in Aqua-MODIS band 1 is found to be $2.2 \%$ in frames 1-338, $1.2 \%$ in frames $339-678,0.9 \%$ in frames 679-1016, and 0.6\% in frames 1017-1354 using DCC. The band 1 RVS changes are also derived using the clear-sky TOA reflectance time-series over the Libya-1 PICS and are found consistent with the DCC results for the matching scan positions. Unlike deserts that are located at fixed geographical locations and are viewed only in certain frames, the DCCs are distributed globally, and therefore, are captured in all MODIS frames, thereby, making DCCs an ideal natural invariant target for RVS characterization. Because the DCC time-series has much less temporal variability compared to the measurements over the desert PICS, RVS characterization using DCCs is more robust and accurate. The DCC approach described in this paper is applicable to other MODIS bands as well and has demonstrated a potential to identify and correct the existing RVS artifacts in the MODIS C6 data. 
This initial study will be expanded to unlock the potential of using DCC to track the sensor RVS over time. Clearly a discontinuity in the DCC mode radiance is evident for frames 1 to 338 during 2011 (Fig. 2). Applying nonlinear trends or piece-wise fits will better capture the RVS drift over time. A sensitivity study is warranted to determine the number of frame increments that DCC will be able to resolve over time. The DCC calibration presented is a large ensemble statistical method, which combines the individual identified DCC pixel level radiances as if they were from a single Earth target. Therefore, the DCC RVS method is capable of providing monthly MODIS RVS assessments, since the sampling across RVS and over time is very uniform. The relative MODIS RVS profile can be estimated monthly and the monthly RVS profile snapshots should be independent of any DCC mode radiance drift over time.

\section{REFERENCES}

[1] Morstad, D. L., David R. Doelling, Rajendra Bhatt, Benjamin Scarino, "The CERES calibration strategy of the geostationary visible channels for CERES cloud and flux products," Proc. SPIE 8153, Earth Observing Systems XVI, 815316 (September 13, 2011).

[2] Minnis, P., L. Nguyen, D. R. Doelling, D. F. Young, W. F. Miller, and D. P. Kratz, 2002: Rapid calibration of operational and research meteorological satellite imagers, Part I: Evaluation of research satellite visible channels as references. J. Atmos. Oceanic Technol., 19, 1233-1249.

[3] Wu, A., X. Xiong, D. R. Doelling, D. L. Morstad, A. Angal, and R. Bhatt, 2013b: Characterization of Terra and Aqua MODIS VIS, NIR, and SWIR spectral band calibration stability. IEEE Trans. Geosci. Remote Sens., 51, 4330-4338.

[4] Doelling, D. R., A. Wu., X. Xiong, B. Scarino, R. Bhatt, C. Haney, and A. Gopalan, 2015a: The radiometric stability and scaling of collection 6 Terra and Aqua-MODIS VIS, NIR, and SWIR spectral bands, IEEE Trans. Geosci. Remote Sens., 53, 4520-4535.

[5] X. Xiong and W. L. Barnes, "An overview of MODIS radiometric calibration and characterization,"Adv. Atmos. Sci., vol. 23, no. 1, pp. 69-79, Jan. 2006.

[6] J. Sun, X. Xiong, A. Angal, H. Chen, A. Wu, and X. Geng, Time-dependent response versus scan angle for MODIS reflective solar bands, IEEE Trans. Geosci. Remote Sens. TGRS.2013.2271448, 2013

[7] H. Chen, X. Xiong and A. Wu, Alternative Approach to Characterize Response versus Scan-angle (RVS) for Reflective Solar Bands, Proceedings of SPIE - Sensors, Systems, and Next-Generation Satellites XV, vol.8176, no.867612 (2011).

[8] D. R. Doelling, D. Morstad, R. Bhatt, and B. Scarino, "Algorithm Theoretical Basis Document (ATBD) for deep convective cloud (DCC) tech- nique of calibrating GEO sensors with Aqua-MODIS for GSICS," GSICS, 2011.

[Online]. Available: http://gsics.nesdis.noaa.gov/pub/Development/

AtbdCentral/GSICS_ATBD_DCC_NASA_2011_09.pdf

[9] Doelling, D. R., D. Morstad, B. R. Scarino, R. Bhatt, and A. Gopalan, 2013: The characterization of deep convective clouds as an invariant calibration target and as a visible calibration technique. IEEE Trans. Geosci. Remote Sens., 51, 1147-1159, doi:10.1109/TGRS.2012.2225066

[10] Doelling, D. R., Nguyen, L. and Minnis, P., 2004, On the use of deep convective clouds to calibrate AVHRR data. In Earth Observing Systems IX, 2-6 August 2004, Denver, CO, Vol. 5542, pp. 281-289 (Bellingham, WA: SPIE).

[11] Doelling, D. R. , G. Hong, D. Morstad, R. Bhatt, A. Gopalan and X. Xiong, The characterization of deep convective cloud albedo as a calibration target using MODIS reflectances, Proc. SPIE 7862, 78620I, 2010).

[12] Bhatt, R., D. R. Doelling, A. Wu, X. Xiaong, B. Scarino, C. O. Haney, and A. Gopalan, 2014: Initial Stability Assessment of S-NPP VIIRS Reflective Solar Band Calibration Using Invariant Desert and Deep Convective Cloud Targets, Remote Sens. Journal, 6, 2809-2826.

[13] Hu, Y. B., B. A. Wielicki, P. Yang, P. W. Stackhouse Jr., B. Lin, and D. F. Young, 2004, “Application of deep convective cloud albedo observation to satellite-based study of the terrestrial atmosphere: Monitoring the stability of spaceborne measurements and assessing absorption anomaly". IEEE Trans. Geosci. Remote Sens., 42, 2594-2599.

[14] Bhatt, R. D.R. Doelling, D. Morstad, B.R. Scarino, A. Gopalan, 2013; Desert-based absolute calibration of successive geostationary visible sensors using a daily exoatmospheric radiance model. IEEE Trans. Geosci. Remote Sens, 52, 3670-3682.

[15] Roujean, J. L., M. J. Leroy, and P. Y. Deschamps, 1992: A bidirectional reflectance model of the earth's surface for the correction of remote sensing data. J. Geophys. Res., 97, 20455-20468. 